\title{
Combination of Circulating Cell-Free DNA and Metabolic Tumor Burden to Distinguish Non-Small Cell Lung Cancer from Tuberculosis
}

\author{
Wen-qiang Zheng \\ Wannan Medical College \\ wannan medical college \\ Bin Quan \\ Wannan Medical College \\ Guang-jian Gao \\ Wannan Medical College \\ Qing Chen \\ Wannan Medical College \\ Yun-hai Dai \\ Wannan Medical College \\ Jian Zhai \\ wannan medical college
}

Puhong Zhang ( $\square$ drzhangpuhong@163.com )

\section{Research}

Keywords: circulating cell-free DNA, 18F-FDG PET/CT, non-small cell lung cancer, tuberculosis, Glucose transporter 1

Posted Date: August 11th, 2020

DOI: https://doi.org/10.21203/rs.3.rs-54830/v1

License: (c) (i) This work is licensed under a Creative Commons Attribution 4.0 International License. Read Full License 


\section{Abstract}

Background: Non-small cell lung (NSCLC) holds high mortality owing to the difficulty to early detection from other lung mass, such as tuberculosis. This study evaluates the clinical value of the combination of circulating cell-free DNA (cfDNA) quantification and metabolic tumor burden to distinguish NSCLC from tuberculosis.

Methods: A total of 149 NSCLC patients, 151 tuberculosis patients and 150 healthy controls were included. Quantifying serum cfDNA fragments from ALU (115 bp) gene by RT-PCR. Metabolic tumor burden (SUV-Max ${ }^{a}$ ) values were detected by preoperative the $18 \mathrm{~F}$-fluorodeoxyglucose positron emission tomography (18F-FDG PET/CT). A549 cell, NCl-H460 cell, NSCLC and tuberculosis mice model were used to elucidate the specific mechanism.

Results: Serum cfDNA levels and SUV-Max ${ }^{a}$ were higher in NSCLC patients than those in healthy controls and those in tuberculosis. Meanwhile, mice models showed the similar discovery. In addition, obvious correlations of cfDNA and metabolic tumor burden were only existed in NSCLC patients and mice model, rather than tuberculosis and control. Moreover, the combination of cfDNA and metabolic tumor burden displayed better effect to distinguish NSCLC from tuberculosis than alone use. Mechanistically, upregulated Glucose transporter 1 (GLU1) increased necroptosis-induce cfDNA rise by FasL/caspase 8/caspase 3 pathway and promoted metabolic tumor burden in NSCLC.

Conclusions: The combination of cfDNA and metabolic tumor burden displayed better effect to distinguish NSCLC from tuberculosis, owing to upregulated GLU1 increased cfDNA levels by FasL/caspase 8/caspase 3 pathways and promoted metabolic tumor burden in NSCLC.

\section{Background}

Lung cancer remains the most common cancer worldwide and is a leading cause of death due to its high morbidity and mortality [1]. NSCLC patients account for approximately $85 \%$ of all lung cancer patients [1]. The majority of NSCLC cases are diagnosed at late stages (stage III or IV) and have systemic tumor metastasis with 5 -year survival rates of $<5 \%$, owing to the low difficulty of early detection of NSCLC from other lung mass, such as tuberculosis[2, 3]. Tuberculosis, a common infectious respiratory disease caused by Mycobacteria, infects $1 / 3$ of the world's population and often radiologically manifests like NSCLC $[2,4]$. In tuberculosis epidemic regions, it is observed that tuberculosis resulted in $58-92 \%$ of false-positive diagnoses of NSCLC and is also listed as one of the major false-positive cases of malignant lymph nodes $[5,6]$. Therefore, there is an increased demand for new diagnostic options based on non-invasive way.

Circulating cell-free DNA (cfDNA) refers to extracellular DNA present in peripheral blood, are mainly derived from tumor DNA fragments by necrotic or apoptotic tumor cells with tumor-epigenetic changes [7, 8]. The activation of caspases $8 /$ caspases 3 pathway, causing apoptosis through promoting poly (ADPribose) polymerase-induced cleavage of DNA, is recognized as best biochemical hallmark of necroptosis 
$[9,10]$. Moreover, size of the cfDNA released from dead lung cancer cells varies between small fragments of 70 to 200 base pairs and large fragments of about $21 \mathrm{~kb}$ and is longer than that of nonneoplastic DNA [11]. Accordingly, the cfDNA quantification is a new diagnostic options for cancer, due to it is a simple, non-invasive access to genetic material detectable in plasma and serum by sensitive molecular biology techniques [12]. Our previous research demonstrates that the levels of cfDNA in patients with NSCLC are obviously higher than that in patients with tuberculosis [13]. Moreover, cfDNA can be used as indicators for identification of NSCLC from tuberculosis [13]. Unfortunately, the sensitivity and specificity of the cfDNA to differentiate NSCLC from tuberculosis was not satisfactory [13].

PET/CT scans with 18F-FDG (a glucose analogue) is recommended for multiple kinds of tumors diagnosis due to its high sensitivity and non-invasive procedure to detect cancer [14, 15]. The metabolic tumor burden is described with the maximum standardized uptake value (SUV-Maxa) of the primary tumor, depending on the rate of 18F-FDG uptake by the tumor $[16,17]$. Currently, PET-CT is a good procedure for the diagnosis of NSCLC patients because it has the capability of identifying the lesion which would pass unnoticed in CT, preventing and reducing unnecessary operation $[18,19]$. However, PETCT shows low specificity for differential diagnosis of NSCLC from tuberculosis, due to the tuberculosis site also presents an elevated level of glucose consumption [20,21]. Of note, recently, a larger prospective, interventional study demonstrated that the combination of cancer blood testing with PET-CT was feasible to screen for multi-cancer and guide intervention [22].

The aim of this study is to evaluate the potential clinical value of the combination of cfDNA and metabolic tumor burden by PET-CT as an auxiliary tool for NSCLC differential diagnosis from tuberculosis in patients with solitary pulmonary nodules. Besides that, NSCLC mice model and tuberculosis mice model are used to verify the correlation of cfDNA and metabolic tumor burden. Finally, gene silencing and inhibitor in vivo and in vitro are performed to elucidate the specific mechanism.

\section{Methods And Materials}

\section{Subjects}

All subjects were recruited from the First and the Second Affiliated Hospital of Wannan Medical College. The protocol for this study was approved by the First Affiliated Hospital of Wannan Medical College and all subjects were provided written informed consent. This research was divided into three groups: healthy controls $(n=150)$, tuberculosis $(n=151)$ and NSCLC $(n=149)$. NSCLC and tuberculosis were diagnosed by pathology through PET/CT-guided biopsy or CT-guided biopsy with World Health Organization criteria $[23,24]$. All subjects were without any anti-tuberculosis or anti-tumor treatment before. Subjects with hepatitis, renal insufficiency, pregnancy, other severe organic disease were excluded in this research.

\section{Animals}

Male BALB/c nude mice (age, 5-6 weeks) were purchased from Slac Laboratory Animal (Shanghai, China). All animal experiments in accordance were with the Laboratory Animal Guidelines for Ethical 
Review of Animal Welfare (GB/T 35892-2018, China) and with approval from the Laboratory Animal Ethics Committee of Wannan Medical College. Mice were randomly allocated into one of seven groups: Control group $(n=8)$, Tuberculosis group $(n=8)$, NSCLC group $(n=8)$; NSCLC+ 0.9\% Saline (NS) group $(n=8)$, NSCLC+ WZB117 group ( $n=8)$, NSCLC+ anti-FasL group $(n=8)$, NSCLC+ Z-IETD-FMK group $(n=8)$.

The mice model of NSCLC was achieved by the injection of human non-small cell lung cancer cells lines A549 cells $(1 \times 106,50 \mu \mathrm{l})$ or $\mathrm{NCl}-\mathrm{H} 460(1 \times 106,50 \mu \mathrm{l})$ via the tail vein. 30 days later, the mice were anesthetized, and the serum and lung tissues were collected [25]. A tuberculosis model was created by the injection of mycobacterium tuberculosis $(1 \times 106 \mathrm{cfu}, 50 \mu \mathrm{l})$ via the tail vein. The sample and tissue were obtained for detection after 7 days [26]. Mices in NSCLC+ WZB117 group, NSCLC+ anti-FasL group, NSCLC+ Z-IETD-FMK group received an injection of WZB117 $(1 \mathrm{mg} / \mathrm{kg})$, anti-FasL antibody (1 ug/mice), Z-IETD-FMK (3 mg/kg) or an equivalent volume of NS via the caudal vein once three days before tissue collection respectively.

\section{Cells}

A549 and $\mathrm{NCl}-\mathrm{H} 460$ cells were cultured in DMEM containing $10 \%$ foetal bovine serum with $5 \% \mathrm{CO} 2$. Cells were allocated into one of four groups: NS-siRNA group ( $n=6)$, GLU1-siRNA group $(n=6)$, FasL-siRNA group $(n=6), Z$-IETD-FMK group $(n=6)$. The siRNA were used to silence GLU1 or FasL gene in A549 and $\mathrm{NCl}-\mathrm{H} 460$ cells as reports $[27,28]$. The rate of cell apoptosis was detected by MTT in the 24th hour.

\section{Materials}

Mycobacterium tuberculosis (ATCC $27294,1 \times 106 \mathrm{cfu} / \mathrm{mg}$ ) was purchased from ATCC. Glu 1 inhibitor (WZB117), caspase-3 and caspase-8 common inhibitor (Z-IETD-FMK) were obtained from MedChemExpress (New Jersey, MJ). Anti-Glu 1, anti-caspase 3 antibody, anti-caspase 8 antibody and anti-FasL antibody were obtained from Abcam (Cambridge, MA). [3H]-2-deoxy-D-glucose was obtained from Perkin Elmer life Sciences and the rate of [3H]-2-deoxy-D-glucose uptake was measured according to the manufacturer's instructions.

\section{Blood collection and DNA isolation}

The serum samples of $2 \mathrm{ml}$ were collected into EDTA-2K containing tubes. The cell-free serum was stored at $-80^{\circ} \mathrm{C}$. Total DNA was extracted from $400 \mu \mathrm{l}$ serum by using QIAamp DNA Blood Mini Kit (Germany. Qiagen). Only the absorbance of the extracted DNA, which was measured by the NanoDrop ND-1000 nucleic acid quantifier (A 260/280 nm), at a ratio of 1.6-1.8 samples were usable.

\section{Measurement of serum cfDNA levels}

The quantization of human and mice serum DNA fragments was detected by quantitative real-time PCR $[13,29]$. Simply, amplifying and quantifying shorter (115 bp) fragments from abundant genomic ALU115 repeats. Sequences of ALU115 primers were forward CCTGAGGTCAGGAGTTCGAG and reverse CCCGAGTAGCTGGGATTACA; Both calibrators and samples were analyzed in triplicate[13]. 


\section{Western blotting}

For western blots, lung tissues were lysed in chilled RIPA buffer diluted in PBS and phosphatase inhibitor for 30 minutes on ice. $10 \mu \mathrm{g}$ of protein was loaded for SDSPAGE and standard western blotting procedures. The following primary antibodies and dilutions were used: GLU1 (1:1000); FasL (1:2000); caspase 3 (1:1000), caspase 8 (1:1000).

\section{PET-CT scan}

PET-CT scan were acquired using integrated PET-CT system (Siemens biograph $\mathrm{mCT}$ ). Images were acquired $1 \mathrm{~h}$ after the administration of $18 \mathrm{~F}-\mathrm{FDG}$. The PET scan was reconstructed by filtered backprojection and ordered-subset expectation-maximization, with data from the CTscan used for attenuation correction. The maximum standardized uptake value (SUV-Max ${ }^{\mathrm{a}}$ ) of the tumor was calculated according to standard formulas. Results were assessed by a nuclear medicine specialist[20].

\section{RNA isolation, microarray analysis and data analysis}

RNAs from the lung tissue were isolated with the RNeasy Mini Kit (Qiagen, Valencia, CA) accordance with the manufacturer's instructions for microarray analysis. Triplicate RNA samples were obtained for each test, and cDNA microarray analysis was performed twice for each RNA sample. Based on KEGG Mapper (https://www.kegg.jp/kegg/tool/map_pathway2.html), the fold changes were analyzed by filtering the data set with P-values $\leq 0.05$ and the signal-to-noise ratio $\geq 2.0$ for screening out differently expressed genes.

\section{Statistical analysis}

Continuous variables were normally distributed by Kolmogorov-Smirnov test and provided as mean \pm SD. Overall comparisons were performed with one-way ANOVA and multiple comparisons between the two groups were derived from the LSD-t test. Categorical variables were expressed as percentages.

Differences in percentages of variables were determined by $\chi 2$ test. Categorical variables were abnormal distribution and shown as Median (Quartile) [M (P25, P75)]. Differences of serum cfDNA concentrations and its integrity and SUV_max a among patients with NSCLC, tuberculosis and healthy controls were analyzed by using the Kruskal-Wallis test. Multiple comparisons between two groups were derived from the Mann Whitney U test. Relationships between cfDNA and SUV_max ${ }^{a}$ were examined using Pearson linear regression analysis. ROC analysis was carried out to determine the AUC, sensitivity and specificity. Statistical analysis was performed by using the SPSS statistical package version 16.0 (Chicago, IL, USA). Statistically significance was established at a $P$ value $<0.05$.

\section{Results}

\section{General conditions}


There were 149 patients with NSCLC, 151 patients with tuberculosis and 150 healthy controls in this research. Characteristics of patients grouped were showed in Table 1. There were no statistical differences in gender, age and comorbidities among these three groups $(P=0.929$ and $P=0.573$, respectively, Table 1). Nevertheless, NSCLC patients showed higher smoking rate (current: $43.62 \%$, former: $36.91 \%$, never: $19.46 \%)$ and tobacco exposure $(42.65 \pm 16.94$ years) compared with healthy controls (current: $30 \%$, former: $26.67 \%$, never: $43.33 \%, P=0.000 ; 30.23 \pm 9.12$ years, $P=0.000$, respectively) and tuberculosis patients (current: $31.13 \%$, former: $39.07 \%$, never: $29.8 \%, P=0.039 ; 36.78 \pm 12.97$ years, $P=$ 0.000 , respectively, Table 1 )

Table 1

The comparison of clinical characteristics among NSCLC, tuberculosis and healthy control.

\begin{tabular}{|lllll|}
\hline & Healthy control $(\mathbf{n = 1 5 0 )}$ & $\begin{array}{l}\text { Tuberculosis } \\
(\mathbf{n = 1 5 1})\end{array}$ & $\begin{array}{l}\text { NSCLC } \\
(\mathbf{n = 1 4 9 )}\end{array}$ & P value \\
\hline Age (years) & $59.92 \pm 8.33$ & $59.27 \pm 9.70$ & $60.08 \pm 9.94$ & 0.734 \\
\hline Gender & & & & 0.932 \\
\hline male & $116(77.33 \%)$ & $114(75.5 \%)$ & $114(76.51 \%)$ & \\
\hline female & $34(22.67 \%)$ & $37(24.5 \%)$ & $35(23.49 \%)$ & \\
\hline Smoking status & & & & $<0.001$ \\
\hline Current & $45(30 \%)$ & $47(31.13 \%)$ & $65(43.62 \%)$ & \\
\hline Former & $40(26.67 \%)$ & $59(39.07 \%)$ & $55(36.91 \%)$ & \\
\hline Never & $65(43.33 \%)$ & $45(29.8 \%)$ & $29(19.46 \%)$ & \\
\hline Tobacco Exposure (years) & $30.23 \pm 9.12$ & $36.78 \pm 12.97$ & $42.65 \pm 16.94$ & $<0.001$ \\
\hline Comorbidities & & & & \\
\hline Hypertension & $55(36.67 \%)$ & $54(35.76 \%)$ & $60(40.27 \%)$ & 0.696 \\
\hline Diabetes & $15(10 \%)$ & $12(7.95 \%)$ & $18(12.08 \%)$ & 0.491 \\
\hline Asthma & 7 & $9(5.96 \%)$ & $12(4.03 \%)$ & 0.473 \\
\hline
\end{tabular}

\section{The cfDNA and SUV-Max ${ }^{\mathrm{a}}$ were increased in NSCLC patients}

The serum level of cfDNA $[19.78(11.52,28.36) \mathrm{ng} / \mu \mathrm{ll}]$ and SUV-Max ${ }^{\mathrm{a}}[3.99(2.33,5.71)]$ were significantly higher in patients with NSCLC than those in healthy controls $[9.75(5.27,13.65) \mathrm{ng} / \mu \mathrm{l}, \mathrm{P}<0.001$, Fig. 1B; $1.93(1.05,2.59), P<0.001$, Fig. $1 C$, respectively] and those in patients with tuberculosis [14.58 (9.149, 18.74) ng/ $\mu \mathrm{l}, \mathrm{P}<0.001$, Fig. 1B; 2.41 (1.50, 3.12), $P<0.001$, Fig. 1C, respectively].

\section{The cfDNA levels were associated with SUV-Max ${ }^{a}$ in NSCLC patients}


In healthy controls and patients with tuberculosis, no statistical association was found between SUVMax ${ }^{a}$ and age $(P=0.319$ and $P=0.102$, respectively), in contrast, statistical association was found in NSCLC patients $(P=0.029$, Table 2). In addition, adenocarcinoma of NSCLC patients showed higher cfDNA and SUV-Max a than those in squamouscarcinoma $[20.93(16.89,36.23) \mathrm{ng} / \mu \mathrm{l}$ vs 17.37 (8.82, $22.81) \mathrm{ng} / \mu \mathrm{l}, \mathrm{P}=0.000 ; 4.36(3.47,8.05)$ vs 3.01 (1.57, 4.22), $\mathrm{P}=0.000$, respectively]. Moreover, patients with advanced NSCLC (pathology stage $\mathbb{X}-\mathbb{Z}$ ) owned higher cfDNA and SUV-Max ${ }^{a}$ than those in early

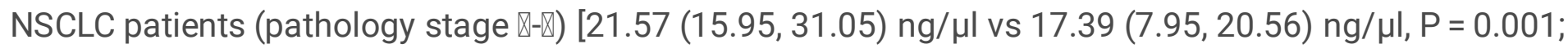
$4.31(3.72,7.95)$ vs $2.89(1.35,3.46), P=0.000$, respectively, Table 2]. Similarly, there is no significant correlation of cfDNA and SUV-Maxa in healthy controls $(r=0.163, P=0.045$, Fig. 1D) and patients with tuberculosis $(r=0.226, P=0.005$, Fig. 1E). However, patients with NSCLC showed obvious correlation of cfDNA and SUV-Max $(r=0.841, P<0.001$, Fig. 1F $)$. 
Table 2

Correlation between clinical characteristics and serum cfDNA or SUVMax ${ }^{\mathrm{a}}$

$$
\mathrm{N} \text { cfDNA }
$$

$\mathrm{K}-\mathrm{W} \times 2 \quad \mathrm{P}$

/Z
SUVMax ${ }^{\mathrm{a}}$
$\mathrm{K}-\mathrm{W} \times 2 \quad P$

/Z

Healthy control

Age (years)

$\leq 65$

119

$9.58(5.06$

0.991

0.319

$1.88(1.14$

0.501

0.479

12.54)

$31 \quad 10.01(7.15$,

17.75)

$1.94(0.89$,

2.71)

Gender (N)

Male

$1169.82(6.48$,

12.53)

$34 \quad 7.14(3.52$,

17.62)

$\begin{array}{lll}-0.608 & 0.543 & 1.90(1.09 \\ & 2.41) \\ & 2.14(.79 \\ & 3.41)\end{array}$

Female

Tuberculosis

Age (years)

$-0.102$

0.918

$-1.448$

0.148

$\leq 65$

$117 \quad 14.59$ (11.34,

17.91)

2.53 (1.69,

3.12)

$>65$

$34 \quad 14.62$ (6.30,

26.99)

$2.10(1.42$,

3.25)

Gender (N)

Male

$114 \quad 14.50$ (9.14,

18.78)

$.43(1.52$

2.99)

Female

$37 \quad 14.66(9.73$,

18.94)

$2.23(1.46$,

5.15)

NSCLC

Age (years)

$-1.636$

0.102

$-2.182$

0.029

$\leq 65$

113

18.64 (14.45,

23.64)

3.53 (2.86,

4.72)

$>65$

$36 \quad 27.90(6.68$ 41.91)

7.19 (2.04,

8.94)

Gender (N)

$-1.110$

0.267

$-1.157$

0.247

Male

114 19.05(11.17, 26.12)

3.81 (2.28, 5.33) 


\begin{tabular}{|c|c|c|c|c|c|c|c|}
\hline & $\mathbf{N}$ & cfDNA & $\begin{array}{l}\mathrm{K}-\mathrm{W} \times 2 \\
/ \mathrm{Z}\end{array}$ & $\mathbf{P}$ & SUVMaxa & $\begin{array}{l}\mathrm{K}-\mathrm{W} \times 2 \\
/ \mathrm{Z}\end{array}$ & $P$ \\
\hline Female & 35 & $\begin{array}{l}20.29(14.29 \\
35.03)\end{array}$ & & & $\begin{array}{l}4.05(3.00, \\
8.30)\end{array}$ & & \\
\hline Pathological type & & & -3.682 & $\begin{array}{l}<.001 \\
0.00\end{array}$ & & -4.080 & $\hat{0.001}$ \\
\hline Adenocarcinoma & 76 & $\begin{array}{l}20.93(16.89 \\
36.23)\end{array}$ & & & $\begin{array}{l}4.36(3.47, \\
8.05)\end{array}$ & & \\
\hline $\begin{array}{l}\text { Squamous } \\
\text { Carcinoma }\end{array}$ & 73 & $\begin{array}{l}17.37(8.82, \\
22.81)\end{array}$ & & & $\begin{array}{l}3.01(1.57, \\
4.22)\end{array}$ & & \\
\hline pathology stage & & & -3.258 & 0.001 & & -5.660 & $\begin{array}{l}<.001 \\
0.00\end{array}$ \\
\hline Stage $\rrbracket-\rrbracket$ & 64 & $\begin{array}{l}17.39(7.95 \\
20.56)\end{array}$ & & & $\begin{array}{l}2.89(1.35, \\
3.46)\end{array}$ & & \\
\hline 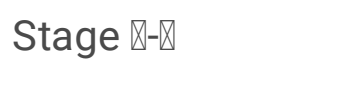 & 85 & $\begin{array}{l}\text { 21.57 (15.95, } \\
31.05)\end{array}$ & & & $\begin{array}{l}4.31(3.72, \\
7.95)\end{array}$ & & \\
\hline
\end{tabular}

\section{Combining cfDNA and SUV-Max ${ }^{a}$ to distinguish NSCLC from healthy controls}

ROC curve analysis showed that the combination of cfDNA and SUV-Max ${ }^{\mathrm{a}}$ (AUC $=0.982, \mathrm{P}=0.000$, cut-off values $=0.22$, sensitivity $=94.5 \%$, specificity $=92.7 \%$ ) displayed higher efficacy to distinguish NSCLC from healthy controls than alone use (AUC $=0.907, P=0.000$, cut-off values $=20.25$, sensitivity $=98.0 \%$, specificity $=67.3 \%$; AUC $=0.901, P=0.000$, cut-off values $=7.67$, sensitivity $=80.6 \%$, specificity $=94.3 \%$, respectively, Fig. 1G, Table 3). 
Table 3

Combining cfDNA and SUVMax ${ }^{a}$ to distinguish NSCLC from healthy controls.

\begin{tabular}{|c|c|c|c|c|c|c|}
\hline & $\begin{array}{l}\text { AUC } \\
\text { ROC }\end{array}$ & $\begin{array}{l}\text { Cut off } \\
\text { value }\end{array}$ & $\begin{array}{l}\text { Sensitivity } \\
(\%)\end{array}$ & $\begin{array}{l}\text { Specificity } \\
(\%)\end{array}$ & $95 \% \mathrm{Cl}$ & $\begin{array}{l}P \\
\text { value }\end{array}$ \\
\hline cfDNA & 0.907 & 20.25 & 98.0 & 67.3 & $\begin{array}{l}0.875- \\
0.939\end{array}$ & $\begin{array}{l}< \\
0.001\end{array}$ \\
\hline SUVMaxa & 0.901 & 7.67 & 80.6 & 94.3 & $\begin{array}{l}0.860- \\
0.941\end{array}$ & $\begin{array}{l}< \\
0.001\end{array}$ \\
\hline $\begin{array}{l}\text { combination of cfDNA and } \\
\text { AUCMaxa }\end{array}$ & 0.982 & 0.22 & 94.5 & 92.7 & $\begin{array}{l}0.971- \\
0.992\end{array}$ & $<.001$ \\
\hline
\end{tabular}

\section{Combining cfDNA and SUV-Maxa to distinguish NSCLC from tuberculosis}

ROC curve analysis showed that the combination of cfDNA and SUV-Max ${ }^{\mathrm{a}}$ (AUC $=0.935, \mathrm{P}=0.000$, cut-off values $=0.46$, sensitivity $=91.9 \%$, specificity $=90.1 \%$ ) also displayed better effect to distinguish NSCLC from tuberculosis than alone use (AUC $=0.804, P=0.000$, cut-off values $=16.83$, sensitivity $=96 \%$, specificity $=65.3 \% ; A U C=0.851, P=0.000$, cut-off values $=3.51$, sensitivity $=67.1 \%$, specificity $=93.7 \%$; respectively, Fig. $1 \mathrm{H}$, Table 4$)$.

Table 4

Combining cfDNA and SUVMax ${ }^{a}$ to distinguish NSCLC from tuberculosis.

\begin{tabular}{|c|c|c|c|c|c|c|}
\hline & $\begin{array}{l}\text { AUC } \\
\text { ROC }\end{array}$ & $\begin{array}{l}\text { Cut off } \\
\text { value }\end{array}$ & $\begin{array}{l}\text { Sensitivity } \\
\text { (\%) }\end{array}$ & $\begin{array}{l}\text { Specificity } \\
\text { (\%) }\end{array}$ & $95 \% \mathrm{Cl}$ & $\begin{array}{l}P \\
\text { value }\end{array}$ \\
\hline cfDNA & 0.804 & 16.83 & 96 & 65.3 & $\begin{array}{l}0.751- \\
0.857\end{array}$ & $\begin{array}{l}< \\
0.001\end{array}$ \\
\hline SUVMaxa & 0.851 & 3.51 & 67.1 & 93.7 & $\begin{array}{l}0.807- \\
0.896\end{array}$ & $\begin{array}{l}< \\
0.001\end{array}$ \\
\hline $\begin{array}{l}\text { combination of cfDNA and } \\
\text { AUCMaxa }\end{array}$ & 0.935 & 0.46 & 91.9 & 90.1 & $\begin{array}{l}0.910- \\
0.960\end{array}$ & $\begin{array}{l}< \\
0.001\end{array}$ \\
\hline
\end{tabular}

The cfDNA was associated with [3H]-2-deoxy-DG uptake in NSCLC mice model 
To investigate the specific mechanism of the relationship between cfDNA and SUV-Maxa ${ }^{a}$, Two NSCLC mice models (A549 and $\mathrm{NCl}-\mathrm{H} 460$ ) and the tuberculosis mice model were used. Metabolic tumor burden was displayed by the value of SUV-Max ${ }^{\mathrm{a}}$ in PET/CT, which depended on the rate of contrast agent (18FFDG) uptake by tumor $[16,17]$. Therefore, the rate of [3H]-2-deoxy-DG uptake was measured to show the metabolic tumor burden in lung tumor of NSCLC mice model. We found A549 NSCLC group and NClH460 NSCLC group both had a higher rate of [3H]-2-deoxy-DG uptake than those in control group and in tuberculosis group (Fig. 2B), similarly to human. Moreover, NSCLC group also showed higher cfDNA level than those in the control group and tuberculosis group (Fig. 2A). Similarly, there is also no correlation of cfDNA and the rate of [3H]-2-deoxy-DG uptake in the control group (Fig. 2C) and tuberculosis group

(Fig. 2D). However, NSCLC group showed obvious association of cfDNA level and [3H]-2-deoxy-DG uptake (Fig. 2E, Fig. 2F).

\section{GLU1/FasL/Caspase-8/Caspase-3 were upregulated in lung tumor tissue of NSCLC mice model}

To further research the specific mechanism of glucose uptake of lung tumor interacting with serum cfDNA, RNA microarray analysis was performed. GLU1, FasL, Caspase-8, caspase-3 gene were significantly upregulated in lung tumor of $\mathrm{A} 549 / \mathrm{NCl}-\mathrm{H} 460 \mathrm{NSCLC}$ group than those in tuberculosis group (Fig. 2G). Besides that, Western blotting also showed that GLU1, FasL, cleaved-caspase 8 and cleavedcaspase 3 were significantly increased, while pro-caspase 8 and pro-caspase 3 were decreased in A549/NCl-H460 NSCLC group than those in tuberculosis group (Fig. $2 \mathrm{H}-\mathrm{I})$.

\section{GLU1 promoted the [3H]-2-deoxy-DG uptake in NSCLC}

To investigate whether [3H]-2-deoxy-DG uptaking rely on GLU1, the GLU 1 inhibitor (WZB117) was used. The increased [3H]-2-deoxy-DG uptake was reversed by WZB117, indicating that the upregulated GLU1 promoted the [3H]-2-deoxy-DG uptake in A549/NCl-H460 NSCLC (Fig. 3C, G).

\section{GLU1 increased cfDNA levels by FasL/caspase 8/caspase 3 pathway in vivo}

To explore whether the necroptosis-induce cfDNA was mediated through GLU1/FasL/caspase 8/caspase 3 pathways, GLU 1 inhibitor (WZB117), anti-FasL antibody, caspase 8 and caspase 3 inhibitor (Z-IETDFMK) were used in vivo. We found that GLU1 increased the expression of cleaved-caspase 3 through increasing FasL and cleaved-caspase 8 expression in lung tumor of A549/NCl-H460 NSCLC mice (Fig. 3A,D,E,H), indicating that GLU1/FasL/caspase 8/caspase 3 pathway was activated in NSCLC to promote necroptosis. Moreover, the increased serum cfDNA was reversed by WZB117, anti-FasL antibody and Z-IETD-FMK (Fig. 3B,F), indicating that GLU1 increased cfDNA levels by FasL/caspase $8 /$ caspase 3 pathways in vivo.

\section{GLU1 promoted apoptosis by FasL/caspase 8/caspase 3 pathway in vitro}

Vitro experiments also demonstrated the similar results in A549 and $\mathrm{NCl}-\mathrm{H} 460$ cells line. Knocking down GLU1 and FasL gene by siRNA and inhibited caspase 8/caspase 3 activities could significantly reduce 
apoptosis, indicated that GLU1 promote apoptosis by FasL/caspase 8/caspase 3 pathway in vitro (Fig. 4).

\section{Discussion}

This study found that the serum levels of cfDNA and metabolic tumor burden (SUV-Max a and [3H]-2deoxy-DG uptake) in patients and mice model of NSCLC were significantly higher than those in healthy controls and tuberculosis. In addition, the cfDNA levels were positively associated with metabolic tumor burden in NSCLC patients and mice model. Moreover, the combination of cfDNA and metabolic tumor burden displayed better effect to distinguish NSCLC patients from tuberculosis patients than alone use. Mechanistically, upregulated GLU1 in tumor tissue promote necroptosis-induce cfDNA rise through FasL/caspase 8/caspase 3 pathways. Meanwhile, upregulated GLU1 also promoted the uptake of glucose of tumor to increase metabolic tumor burden in NSCLC.

Several reports have demonstrated higher levels of cfDNA in the serum of lung cancer patients compared with healthy controls, serving as a potential diagnostic biomarker [13,30,31]. In this study, both NSCLC patients and mice model displayed higher levels of cfDNA than those in healthy controls, indicating that cfDNA could be used as a potential diagnostic biomarker for NSCLC. In addition, our present research also demonstrated that the cfDNA levels also increased in NSCLC patients and mice model compared with those in tuberculosis patients and mice model, elucidating that cfDNA was a potential diagnostic biomarker to distinguish NSCLC from tuberculosis just as our previous report [13]. Unfortunately, the sensitivity and specificity of the cfDNA to distinguish NSCLC from tuberculosis were not satisfactory, therefor, it was necessary to seek a new approach to improve the efficiency of the differential diagnosis.

18F-FDG PET/CT was a valuable new imaging modality for diagnosis and differential diagnosis of NSCLC $[32,33]$. PET/CT parameters (i.e., SUV-Maxa) could provide useful data on tumor metabolism. For instance, the SUV-Maxa ${ }^{\text {a }}$, the value of a single voxel with the highest radiotracer (18F-FDG) concentrations within the tumor, was a indicator of the metabolic tumor burden $[16,17]$. This study found that SUV-Max ${ }^{a}$ was increased in NSCLC patients compared with that in healthy controls and tuberculosis patients, and could be used for the diagnosis and the differential diagnosis of NSCLC just as a previous report [6]. Of note, although SUV-Maxa was useful and might be a surrogate marker of the metabolic tumor burden, it was affected by many factors and was highly sensitive to noise [34, 35]. A previous study has demonstrated that SUV-Max ${ }^{a}$ had low sensitivity for distinguishing NSCLC from tuberculosis [20]. This study also revealed this result, indicating that a combined approach to improve the differential diagnosis rate of NSCLC from tuberculosis was required.

Recently, a larger prospective, interventional study demonstrated that the combination of blood testing with PET-CT could be used to screen for lung cancer [22]. Therefore, the combination of cfDNA and metabolic tumor burden was performed to distinguish NSCLC from tuberculosis. Intriguingly, ROC curve analysis showed that the combination of cfDNA and SUV-Max displayed better effect to distinguish NSCLC from tuberculosis than alone use. Furthermore, patients with NSCLC, rather than healthy controls 
or patients with tuberculosis, showed obvious positive correlation of cfDNA and SUV-Maxa . In addition, this association also existed in the NSCLC mice model, rather than control mice model or the tuberculosis mice model, indicating that there may be a connection, to a certain degree, between the metabolism of cfDNA and tumor burden.

Tumor cell adapted to hypoxic conditions by controlling the expression of many endogenous glycolysisrelated transporters and enzymes, such as GLU1, which played a pivotal role in the mechanisms of cellular metabolism [36, 37]. Although several studies have reported a correlation between GLU1 expression and SUV-Max ${ }^{\mathrm{a}}$ in NSCLC patients [17, 38], none have verified this relationship in an animal model. This research found that the expression of GLU1 mRNA and protein were also increased markedly in two NSCLC mice model, similarly as NSCLC patients, moreover, the increased glucose uptake in tumor cell was reversed by a GLU1-specific inhibitor, indicating that upregulated GLU1 increased metabolic tumor burden (SUV-Maxa) by promoting the glucose uptake in NSCLC.

Deregulation in apoptotic cell death machinery was a hallmark of cancer, and necroptosis alteration was responsible for the tumor burden and development and progression $[9,10]$. Circulating cfDNA were mainly derived from tumor DNA fragments by apoptotic tumor cells, while necroptosis was stimulated by the activation of caspases 8 and caspases $3[7,8]$. Our present research found that upregulated GLU1 increased the expression of cleaved-caspases 8 and cleaved-caspase 3 through upregulating FasL to promote necroptosis in vivo and in vitro. Moreover, increased serum cfDNA levels were reversed by GLU1specific inhibitor, anti-FasL antibody and caspases 8 and caspase 3 common inhibitor, indicating that the increased serum cfDNA depended on GLU1/ FasL/caspase 8/caspase 3 pathway in NSCLC.

This study has several limitations. First, the sample size was not enough to Distinguish NSCLC from tuberculosis convincingly by the combination of cfDNA and metabolic tumor burden. Therefore, any comprehensive analysis of this issue would need to be specifically addressed in a case-control matched study with considerably larger cohorts. Second, other mechanisms for the correlation of cfDNA and metabolic tumor burden, except for GLU1/ FasL/caspase 8/caspase 3 pathways, should be considered.

\section{Conclusion}

As shown in Fig. 5, the present study demonstrated that serum levels of cfDNA and metabolic tumor burden in patients and mice model of NSCLC were significantly increased than those in healthy controls and tuberculosis. Moreover, serum cfDNA levels were positive correlated with metabolic tumor burden in NSCLC. In addition, the combination of cfDNA and metabolic tumor burden displayed better effect to distinguish NSCLC patients from tuberculosis patients than alone use. Mechanistically, upregulated GLU1 increased the serum levels of cfDNA by FasL/caspase 8/caspase 3 pathways and promoted the uptake of glucose to raise metabolic tumor burden in NSCLC.

\section{Abbreviations}


NSCLC: non-small cell lung cancer; cfDNA: cell-free DNA; 18F-FDG PET/CT:18F-fluorodeoxyglucose positron emission tomography ; SUV-Maxa: the maximum standardized uptake value; AUC: area under the curve; GLU1:Glucose transporter 1.

\section{Declarations}

\section{Acknowledgement}

The authors of this study would like to acknowledge the contributions of study participants.

\section{Funding}

This study was supported by grants from the National Natural Science Foundation of China (81200632), as well as the Natural Science Research Project of Anhui Colleges and Universities (KJ2016A737).

\section{Author contributions}

W-Q Zheng and B Quan and P-H Zhang conceived and carried out experiments, P-H Zhang and W-Q Zheng and B Quan conceived experiments and analysed data. G-J Gao and Q Chen and H-Y Dai and J Zhai carried out experiments. P-H Zhang write the paper and had final approval of the submitted and published versions.

\section{Data availability statement}

Research data are stored in an institutional repository and will be shared upon request to the corresponding author.

\section{Ethics approval and consent to participate}

This study was reviewed and approved by the Medical Ethics Committee of the First Affiliated Hospital of Wannan Medical College, Wuhu, China. All animal experiments in accordance were with the Laboratory Animal Guidelines for Ethical Review of Animal Welfare (GB/T 35892-2018, China) and with approval from the Laboratory Animal Ethics Committee of Wannan Medical College.

\section{Consent for publication}

All authors have agreed to publish this manuscript.

\section{Conflict of Interest}

The all authors declare that they have no conflict of interest.

\section{References}

1. Siegel R L, and Miller K D. Cancer statistics, 2020.2020; 70: 7-30. 
2. Reck M, and Rabe K F. Precision Diagnosis and Treatment for Advanced Non-Small-Cell Lung Cancer. N Engl J Med.2017; 377: 849-861.

3. RS L, SS L, YM T, HC W, CC L, and JY L. Diagnostic value of transbronchial lung biopsy under fluoroscopic guidance in solitary pulmonary nodule in an endemic area of tuberculosis. Respiratory medicine.1996; 90: 139-143.

4. Schrager L K, Vekemens J, Drager N, Lewinsohn D M, and Olesen O F. The status of tuberculosis vaccine development. Lancet Infect Dis.2020; 20: e28-e37.

5. Shim S S, Lee K S, Kim B T, Chung M J, Lee E J, Han J, et al. Non-small cell lung cancer: prospective comparison of integrated FDG PET/CT and CT alone for preoperative staging. Radiology.2005; 236 : 1011-1019.

6. Kang F, Wang S, Tian F, Zhao M, Zhang M, Wang Z, et al. Comparing the Diagnostic Potential of 68Ga-Alfatide II and 18F-FDG in Differentiating Between Non-Small Cell Lung Cancer and Tuberculosis. J Nucl Med.2016; 57: 672-677.

7. Chabon $\mathrm{J} \mathrm{J}$, and Hamilton E G. Integrating genomic features for non-invasive early lung cancer detection2020; 580: 245-251.

8. De Mattos-Arruda L, Mayor R, Ng C K Y, Weigelt B, Martinez-Ricarte F, Torrejon D, et al. Cerebrospinal fluid-derived circulating tumour DNA better represents the genomic alterations of brain tumours than plasma. Nat Commun.2015; 6: 8839.

9. Bock F J, and Tait S W G. Mitochondria as multifaceted regulators of cell death2020; 21: 85-100.

10. Singh R, Letai A, and Sarosiek K. Regulation of apoptosis in health and disease: the balancing act of BCL-2 family proteins. Nat Rev Mol Cell Biol.2019; 20: 175-193.

11. Esposito A, Criscitiello C, Trapani D, and Curigliano G. The Emerging Role of "Liquid Biopsies," Circulating Tumor Cells, and Circulating Cell-Free Tumor DNA in Lung Cancer Diagnosis and Identification of Resistance Mutations. Curr Oncol Rep.2017; 19: 1.

12. E H, L A, and MR S. Cell-Free DNA and Apoptosis: How Dead Cells Inform About the Living. Trends in molecular medicine.2020; 26: 519-528.

13. Leng $\mathrm{S}$, Zheng J, Jin $\mathrm{Y}$, Zhang H, Zhu Y, Wu J, et al. Plasma cell-free DNA level and its integrity as biomarkers to distinguish non-small cell lung cancer from tuberculosis. Clin Chim Acta.2018; 477: 160-165.

14. Creff G, Devillers A, Depeursinge A, Palard-Novello X, Acosta O, Jegoux F, et al. Evaluation of the Prognostic Value of FDG PET/CT Parameters for Patients With Surgically Treated Head and Neck Cancer: A Systematic Review. JAMA Otolaryngol Head Neck Surg.2020.

15. De Leyn P, Dooms C, Kuzdzal J, Lardinois D, Passlick B, Rami-Porta R, et al. Revised ESTS guidelines for preoperative mediastinal lymph node staging for non-small-cell lung cancer. Eur $\mathrm{J}$ Cardiothorac Surg.2014; 45: 787-798.

16. F N, J W, C L, L D, J X, and Y L. Primary tumor standardized uptake value measured on F18Fluorodeoxyglucose positron emission tomography is of prediction value for survival and local control in non-small-cell lung cancer receiving radiotherapy: meta-analysis. Journal of thoracic 
oncology : official publication of the International Association for the Study of Lung Cancer.2014; 9: 834-842.

17. YW K, SJ L, and SY P. Differential expression and prognostic significance of GLUT1 according to histologic type of non-small-cell lung cancer and its association with volume-dependent parameters. Lung cancer (Amsterdam, Netherlands).2017; 104: 31-37.

18. Vella M, Meyer C S, Zhang N, Cohen B E, Whooley M A, Wang S, et al. Association of Receipt of Positron Emission Tomography-Computed Tomography With Non-Small Cell Lung Cancer Mortality in the Veterans Affairs Health Care System. JAMA Netw Open.2019; 2: e1915828.

19. Pacilio M, Lauri C, Prosperi D, Petitti A, and Signore A. New SPECT and PET Radiopharmaceuticals for Imaging Inflammatory Diseases: A Meta-analysis of the Last 10 Years. Semin Nucl Med.2018; 48: 261-276.

20. Werutsky G, Hochhegger B, Lopes de Figueiredo Pinto J A, Martinez-Mesa J, Zanini M L, Berdichevski $\mathrm{E} \mathrm{H}$, et al. PET-CT has low specificity for mediastinal staging of non-small-cell lung cancer in an endemic area for tuberculosis: a diagnostic test study (LACOG 0114). BMC Cancer.2019; 19: 5.

21. G W, B H, JA L d F P, J M-M, ML Z, EH B, et al. PET-CT has low specificity for mediastinal staging of non-small-cell lung cancer in an endemic area for tuberculosis: a diagnostic test study (LACOG 0114). BMC cancer.2019; 19: 5.

22. AM L, AH B, I K, A W, A H, AT C, et al. Feasibility of blood testing combined with PET-CT to screen for cancer and guide intervention. Science (New York, NY).2020. https://doi.org/10.1038/sj.jhh.1002269.

23. Zumla A, George A, Sharma V, Herbert R H, Oxley A, and Oliver M. The WHO 2014 global tuberculosis report-further to go. Lancet Glob Health.2015; 3: e10-12.

24. Travis W D, Brambilla E, Nicholson A G, Yatabe Y, Austin J H M, Beasley M B, et al. The 2015 World Health Organization Classification of Lung Tumors: Impact of Genetic, Clinical and Radiologic Advances Since the 2004 Classification. J Thorac Oncol.2015; 10: 1243-1260.

25. Zhang X, Liu Y, Peng X, Zeng Y, Li L, Wang J, et al. Influence of the vaccinating density of A549 cells on tumorigenesis and distant organ metastasis in a lung cancer mice model. Cell Mol Biol (Noisy-legrand).2018; 64: 53-57.

26. Vilcheze C, Kim J, and Jacobs W R, Jr. Vitamin C Potentiates the Killing of Mycobacterium tuberculosis by the First-Line Tuberculosis Drugs Isoniazid and Rifampin in Mice. Antimicrob Agents Chemother.2018; 62.

27. Y Y, HJ K, M Z, P M, M N, BS K, et al. Glucose-linked sub-50-nm unimer polyion complex-assembled gold nanoparticles for targeted siRNA delivery to glucose transporter 1-overexpressing breast cancer stem-like cells. Journal of controlled release : official journal of the Controlled Release Society.2019; 295: 268-277.

28. C Z, F G, F T, and M Z. Fas/FasL Complex Promotes Proliferation and Migration of Brain Endothelial Cells Via FADD-FLIP-TRAF-NF-KB Pathway. Cell biochemistry and biophysics.2015; 71: 1319-1323.

29. LA A, AV S k, MA Z, and NL M. Targeting Circulating SINEs and LINEs with DNase I Provides Metastases Inhibition in Experimental Tumor Models. Molecular therapy Nucleic acids.2020; 20: 50- 
61.

30. H Z, X W, J Y, S W, Z L, and C Y. Clinical applications of liquid biopsies for early lung cancer detection. American journal of cancer research.2019; 9: 2567-2579.

31. KM K, MB M, S D, P P, M T, and TA K. Tumor-specific genetic aberrations in cell-free DNA of gastroesophageal cancer patients. Journal of gastroenterology.2019; 54: 108-121.

32. M K, B P, G S, A M, A G, E S, et al. CT, [F]FDG-PET/CT and clinical findings before and during early Covid-19 onset in a patient affected by vascular tumour. European journal of nuclear medicine and molecular imaging. 2020.

33. J V, BM F, C D, and J M. Positron-emission tomography in prognostic and therapeutic assessment of lung cancer: systematic review. The Lancet Oncology.2004; 5: 531-540.

34. Park S Y, Cho A, Yu W S, Lee C Y, Lee J G, Kim D J, et al. Prognostic value of total lesion glycolysis by 18F-FDG PET/CT in surgically resected stage IA non-small cell lung cancer. J Nucl Med.2015; 56: 4549.

35. Hyun S H, Ahn H K, Kim H, Ahn M J, Park K, Ahn Y C, et al. Volume-based assessment by (18)F-FDG $\mathrm{PET} / \mathrm{CT}$ predicts survival in patients with stage III non-small-cell lung cancer. Eur J Nucl Med Mol Imaging.2014; 41: 50-58.

36. Wang J, Yu J, Zhang Y, Kahkoska A R, Wang Z, Fang J, et al. Glucose transporter inhibitor-conjugated insulin mitigates hypoglycemia. Proc Natl Acad Sci U S A.2019; 116: 10744-10748.

37. Rosell R, Bivona T G, and Karachaliou N. Genetics and biomarkers in personalisation of lung cancer treatment. Lancet.2013; 382: 720-731.

38. Kaira K, Serizawa M, Koh Y, Takahashi T, Yamaguchi A, Hanaoka H, et al. Biological significance of 18F-FDG uptake on PET in patients with non-small-cell lung cancer. Lung Cancer.2014; 83: 197-204.

\section{Figures}



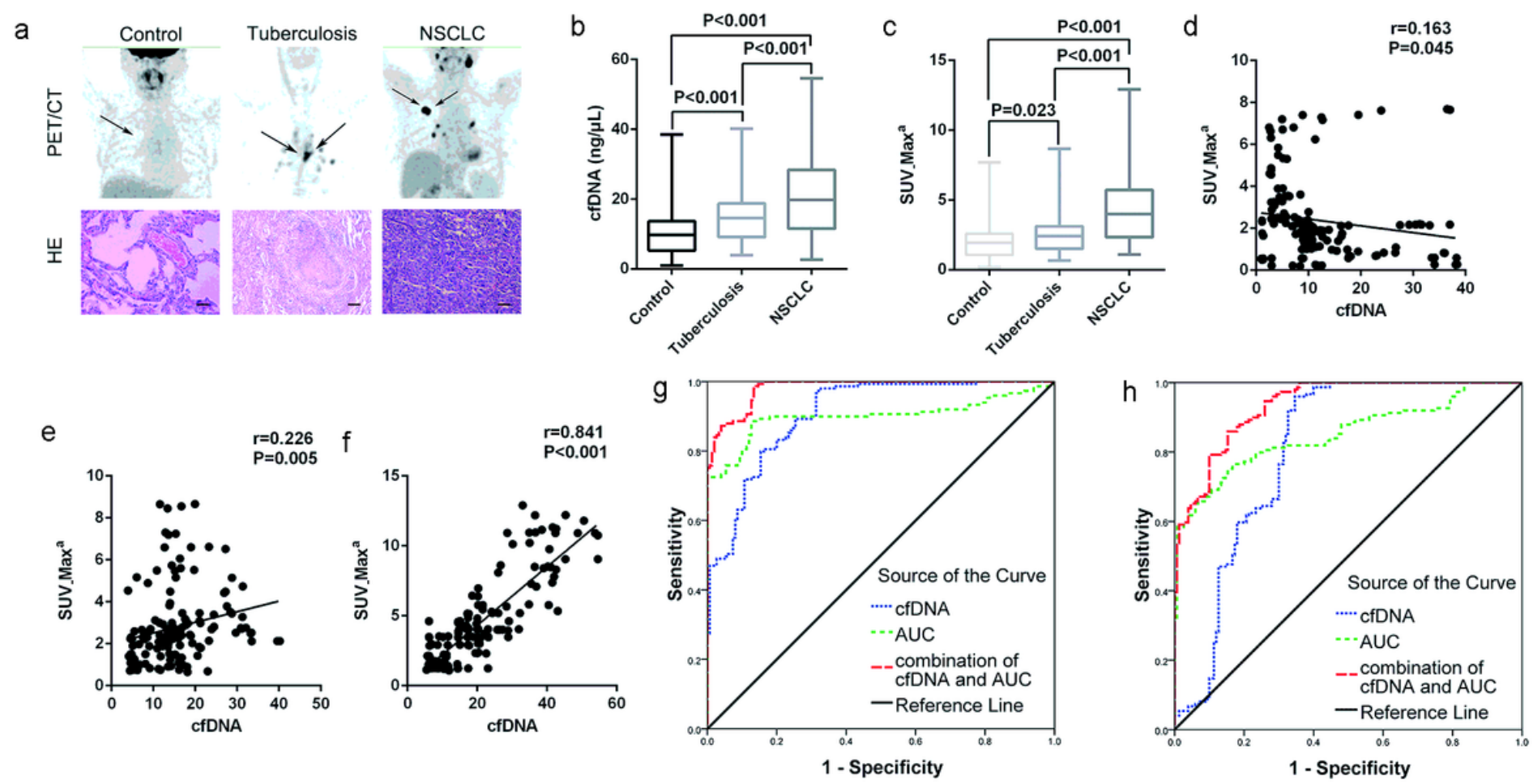

Figure 1

The combination of cfDNA and SUV-Maxa displayed better effect to distinguish NSCLC from tuberculosis. (a) The metabolic tumor burden was described with the maximum standardized uptake value (SUV-Maxa) of the primary tumor, measured by PET/CT. NSCLC and tuberculosis were diagnosed by pathology through PET/CT-guided biopsy or CT-guided biopsy. (b) Serum cfDNA levels in healthy controls, tuberculosis patients and NSCLC patients. (c) The metabolic tumor burden (SUV-Maxa) in healthy controls, tuberculosis patients and NSCLC patients. (d) The correlation of cfDNA and SUV-Maxa in healthy controls. (e) The correlation of cfDNA and SUV-Maxa in tuberculosis patients. (f) The correlation of cfDNA and SUV-Maxa in NSCLC patients. (g) ROC curve analysis to distinguish NSCLC from healthy controls by the serum cfDNA levels, SUV-Maxa or the combination of cfDNA and SUV-Maxa. (h) ROC curve analysis to distinguish NSCLC from tuberculosis by the serum cfDNA levels, SUV-Maxa or the combination of cfDNA and SUV-Maxa. Values represent means (Quartile) [M (P25, P75)]. 
a

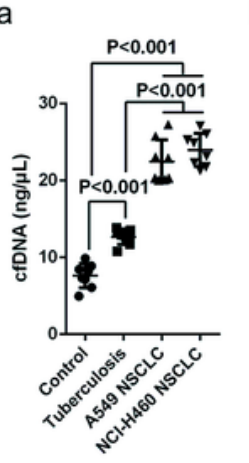

g

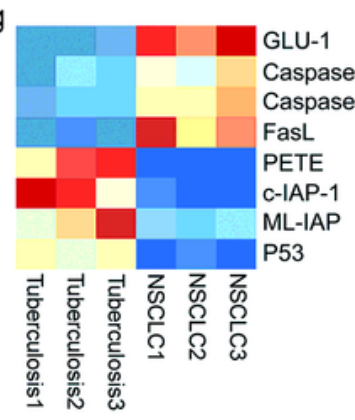

j

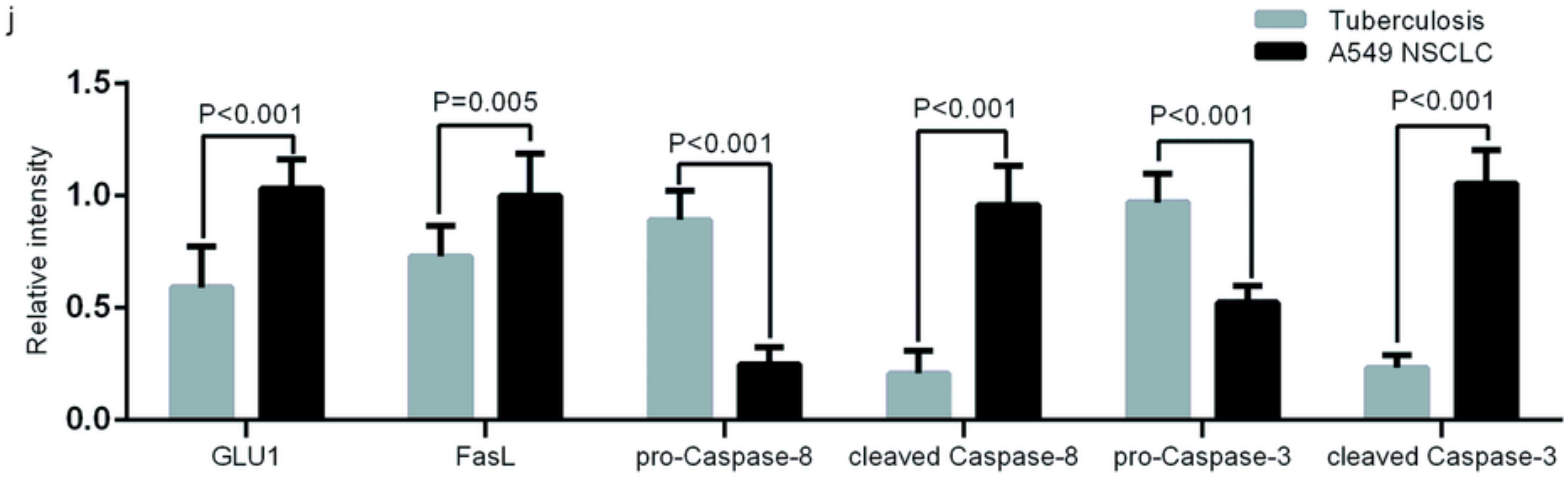

b $\quad P<0.001 \quad$ C
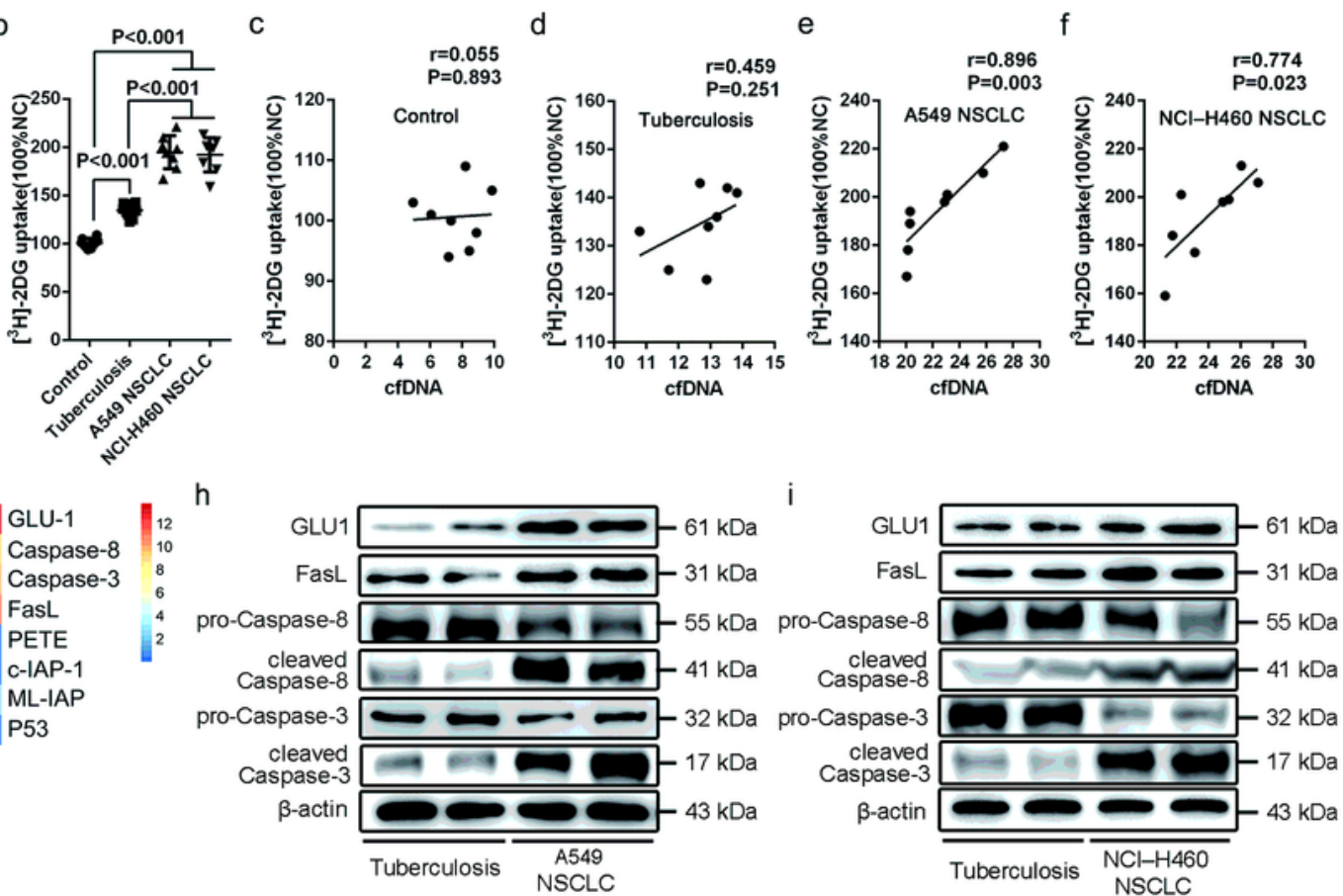

$\mathrm{k}$

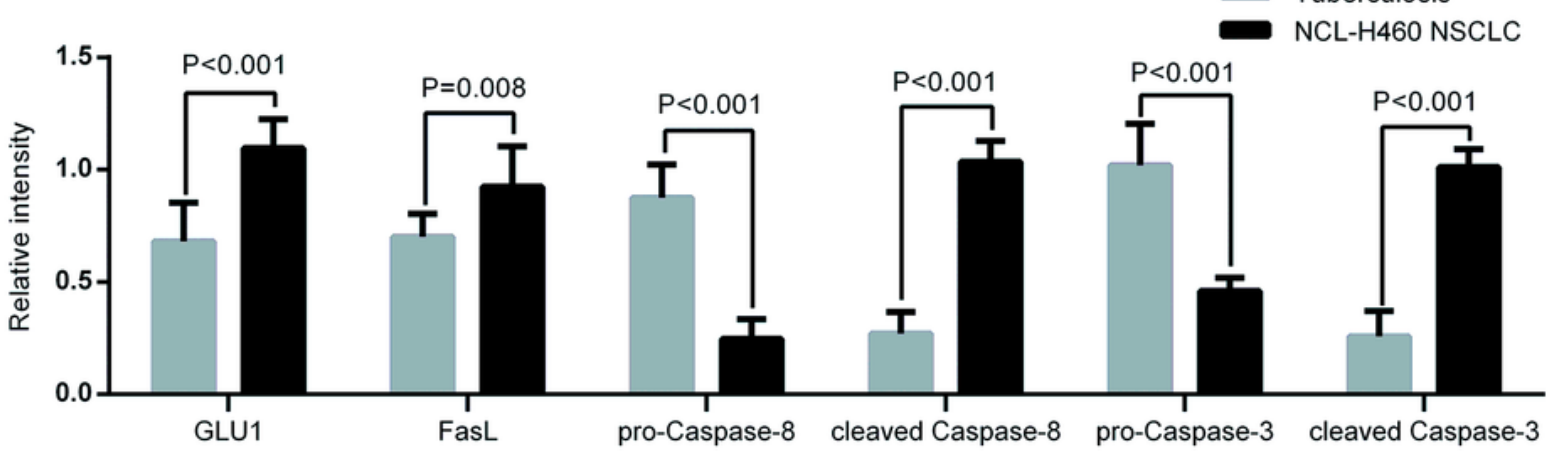

Figure 2

Serum cfDNA levels were correlated with [3H]-2-deoxy-DG uptake in NSCLC mice model (a) Serum cfDNA levels in control mice model, tuberculosis mice model and NSCLC mice model. (b) The metabolic tumor burden ([3H]-2-deoxy-DG uptake). (c) The correlation of cfDNA and [3H]-2-deoxy-DG uptake in controls. (d) The correlation of cfDNA and [3H]-2-deoxy-DG uptake in tuberculosis mice model. (e) The correlation of cfDNA and [3H]-2-deoxy-DG uptake in A549 NSCLC mice model. (f) The correlation of cfDNA and [3H]-2- 
deoxy-DG uptake in NCl-H460 NSCLC mice model. (g) RNA microarray analysis was performed to investigate the differential gene between NSCLC mice model and tuberculosis mice model. (h, j) GLUT1, FasL, pro-caspase 8, cleaved caspase 8, pro-caspase 3 and cleaved caspase 3 expression was evaluated using Western blotting in tuberculosis mice model and A549 NSCLC mice model. (i, k) GLUT1, FasL, procaspase 8 , cleaved caspase 8, pro-caspase 3 , cleaved caspase 3 expression was evaluated using Western blotting in tuberculosis mice model and $\mathrm{NCl}-\mathrm{H} 460 \mathrm{NSCLC}$ mice model.(Values represent mean $\pm \mathrm{SD}, \mathrm{n}=8$ per group).

a

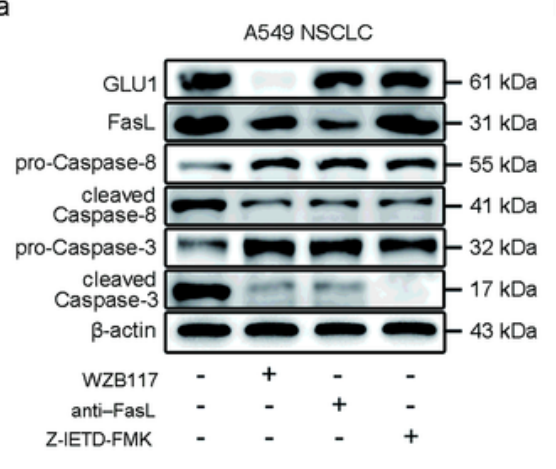

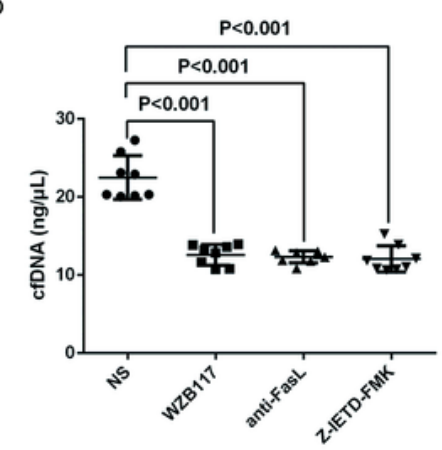

c

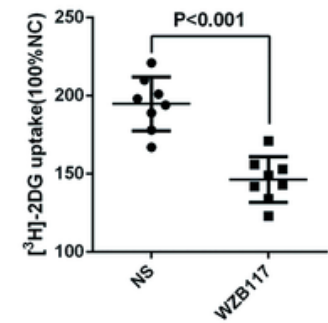

d

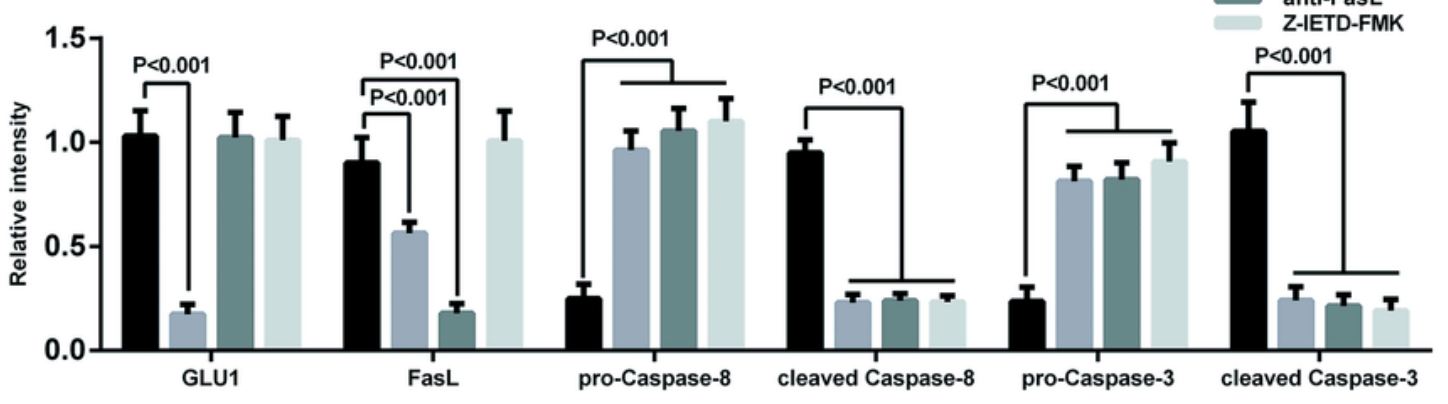

e

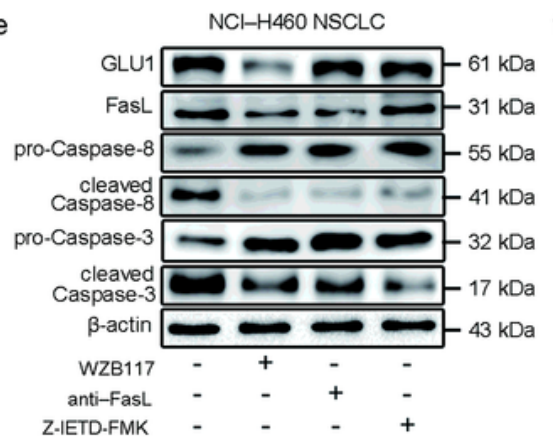

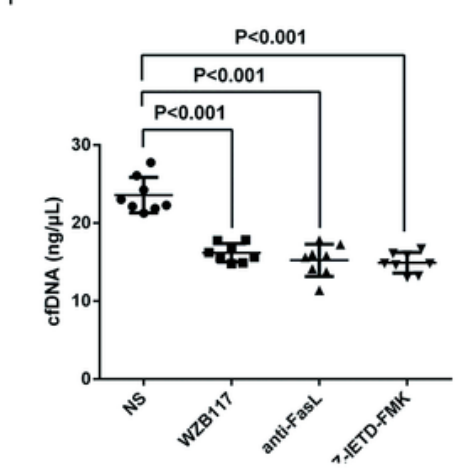

g

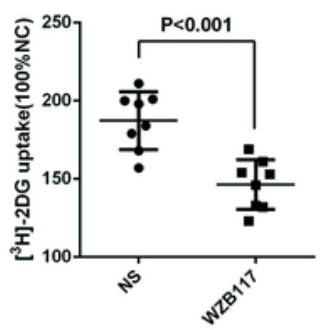

h

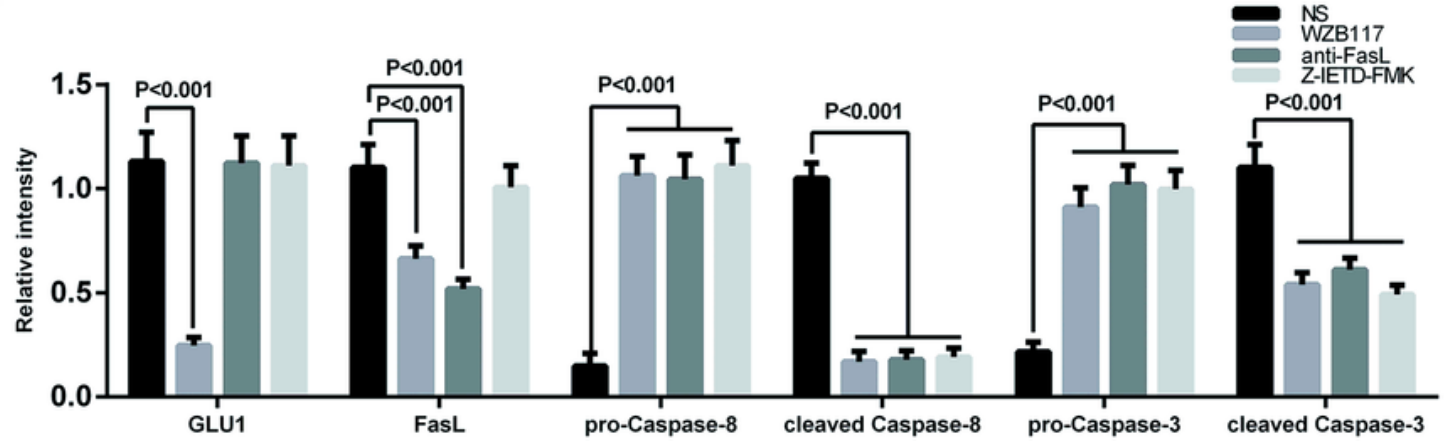




\section{Figure 3}

GLU1 increased cfDNA levels by FasL/caspase 8/caspase 3 pathway and promoted the [3H]-2-deoxy-DG uptake in vivo Glu 1 Inhibitor (WZB117), anti-FasL antibody, caspase-3 and caspase-8 common inhibitor (Z-IETD-FMK) were injected into (a, d) A549 NSCLC mice model or $(e, h)$ NCl-H460 NSCLC mice model via the caudal vein once three days to elucidate the specific signal pathway. GLUT1, FasL, pro-caspase 8, cleaved caspase 8 , pro-caspase 3 , cleaved caspase 3 expression was evaluated using Western blotting. Increased serum cfDNA levels were reversed by anti-FasL antibody and Z-IETD-FMK in (b) A549 NSCLC mice model or (f) NCl-H460 NSCLC mice model. The increased [3H]-2-deoxy-DG uptake was reversed by WZB117 in (c) A549 NSCLC mice model or (g) NCl-H460 NSCLC mice model. (Values represent mean $\pm S D, n=8$ per group).

a

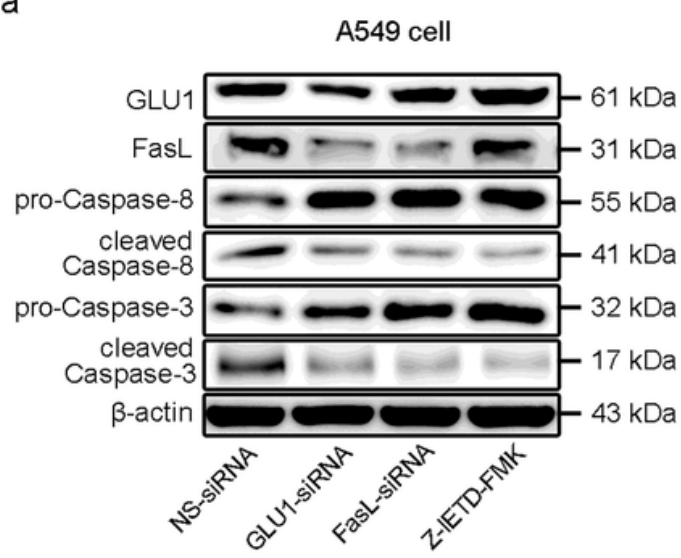

d

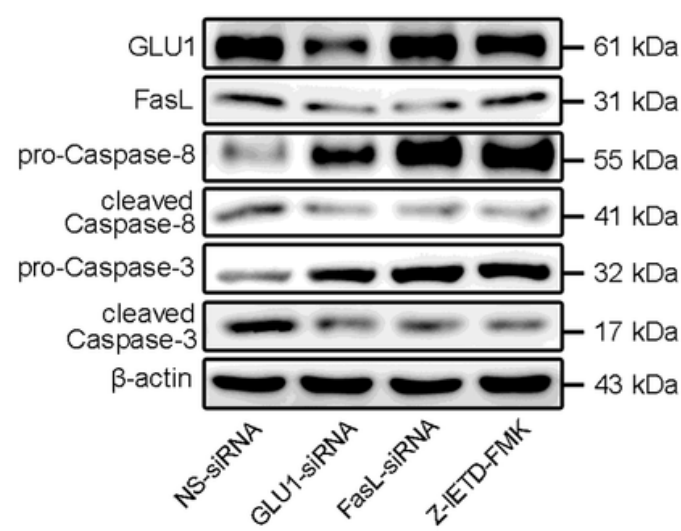

b

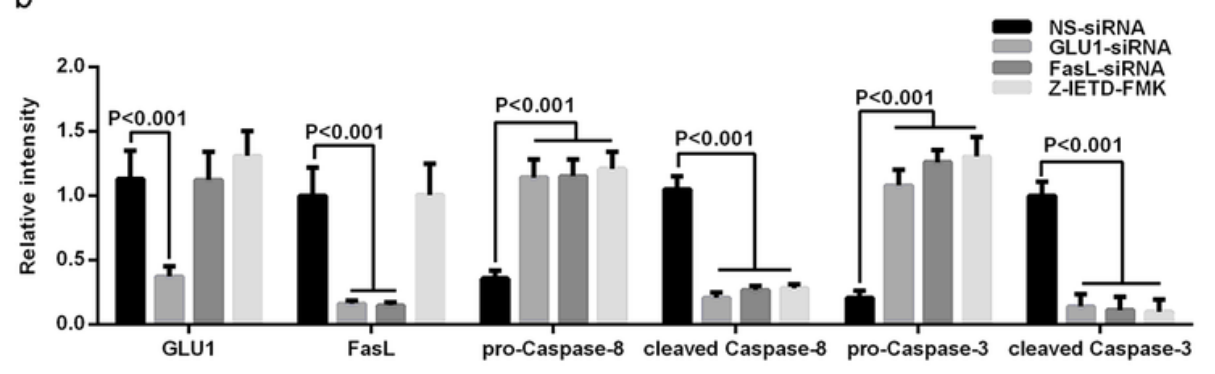

C

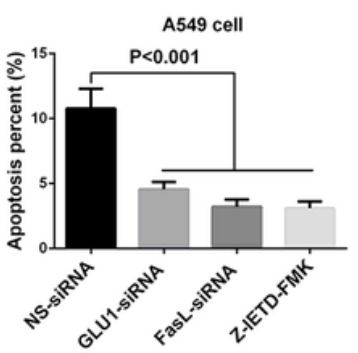

f

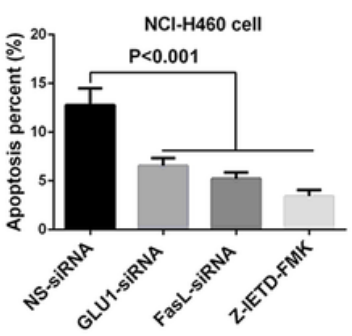

e

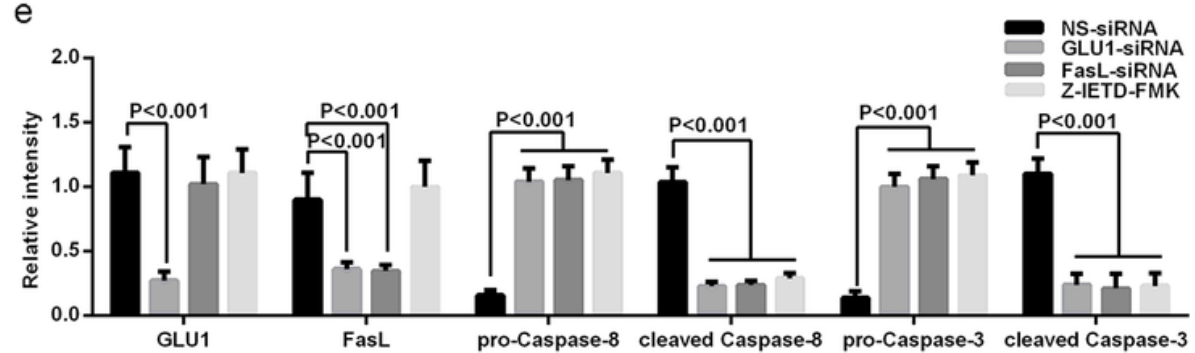

Figure 4

GLU1 promoted apoptosis by FasL/caspase 8/caspase 3 pathway in vitro The GLU1 or FasL gene were knocked down by siRNA and the activation of caspase-3 and caspase-8 were blocked by Z-IETD-FMK. GLUT1, FasL, pro-caspase 8, cleaved caspase 8, pro-caspase 3, cleaved caspase 3 expression was evaluated by Western blotting in (a, b) A549 cell and (d, e) NCl-H460 cell. Apoptosis percent of (c) A549 cell and (f) $\mathrm{NCl}-\mathrm{H} 460$ cell were detected by MTT in the 24th hour. (Values represent mean $\pm S D, n=6$ per group). 


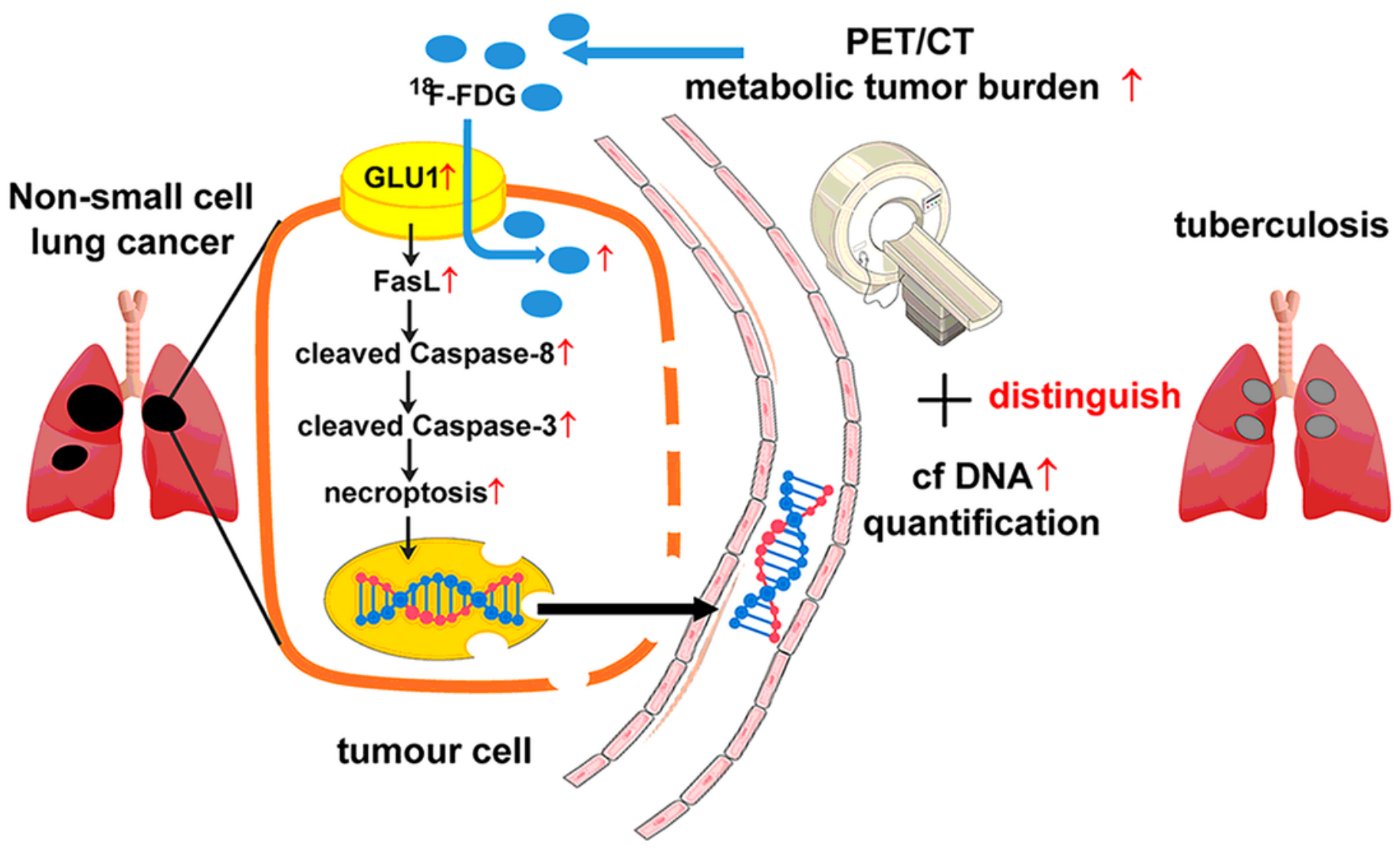

Figure 5

Summary, the combination of cfDNA and metabolic tumor burden displayed better effect to distinguish NSCLC from tuberculosis, owing to upregulated GLU1 in tumor tissue promote necroptosis-induce cfDNA release through FasL/caspase 8/caspase 3 pathway, as will as also promoted the uptaking of glucose of tumor to increase metabolic tumor burden in NSCLC. 